\title{
Near infrared whole body imaging
}

\author{
Martha Hale \\ Toxinology Division, \\ US Army Medical Research Institute of Infectious Diseases, \\ Ft. Detrick, MD 21702, USA \\ E-mail: martha.hale@us.army.mil
}

\begin{abstract}
Near infrared imaging (NIR), using dyes that emit between 700 and $900 \mathrm{~nm}$, overcome many of the absorption and light scatter problems that have been associated with whole body and tissue imaging. This review summarises current areas in which NIR provides an excellent non-invasive technology for tracking and investigating molecular movement in vivo.
\end{abstract}

Keywords: near infrared imaging; NIR; in vivo imaging botulinum neurotoxin.

Reference to this paper should be made as follows: Hale, M. (2012) 'Near infrared whole body imaging', The Botulinum J., Vol. 2, No. 2, pp.168-172.

Biographical notes: Martha Hale received her $\mathrm{PhD}$ (Microbiology) from the Virginia Commonwealth University-Health Sciences Division, Richmond, VA and post-doctoral training at the National Institutes of Health, Bethesda, MD. She has performed research in various fields that are important in bioterrorism defence. Recently, she has developed near infrared imaging to study movement of biological toxins in vitro and in vivo. Her current research is focused upon development of therapeutics for various select agent toxins. She is a member of the American Association of Immunologists and American Society for Microbiology.

Over the last several years, optical-based imaging has been used to visualise bacterial localisation and tissue damage in vivo. Although excellent for cellular or molecular imaging, fluorophores that are activated and then emit in the visible spectrum do not have the best characteristics for whole body imaging. In vivo optical imaging depends upon the specific characteristics of photons and the photon's capability to transmit fluorescence through tissue (Frangioni, 2003). The ability to detect photon movement within living tissue depends upon three processes: light absorption, light scatter, and fluorescent emissions.

For whole body imaging, maximising the depth of tissue penetration is extremely important (Licha and Olbrich, 2005; Olive, 2006). Photon absorption in tissue is primarily due to deoxy-hemaglobin and oxy-hemoglobin, although other compounds such as melanin and lipids also contribute, making absorption and light scatter a limiting factor in tissue penetration. Absorption and scatter tend to decrease with increasing wavelengths so that photons with spectral emissions below $700 \mathrm{~nm}$ can only penetrate a few centimetres, certainly not the depth required for whole-body imaging. Between 700 and $900 \mathrm{~nm}$, the NIR region, tissue absorption is at its lowest. When the absorption coefficients are significantly smaller, light penetrates more deeply and the signal can be visualised several centimetres in depth. At wavelengths greater than $900 \mathrm{~nm}$, water constitutes the primary absorbance problem and detections above $900 \mathrm{~nm}$ are again less 
distinct (Figure 1). In addition to light absorption, animal tissues emit fluorescence throughout the visible range, and detection below $700 \mathrm{~nm}$ becomes obscured by the tissue's auto-fluorescence. Low tissue auto-fluorescence combined with high light penetration make NIR an excellent technology for in vivo imaging.

Figure 1 The absorption spectra of oxygenated and deoxygenated hemoglobin and water for typical concentrations found in the brain

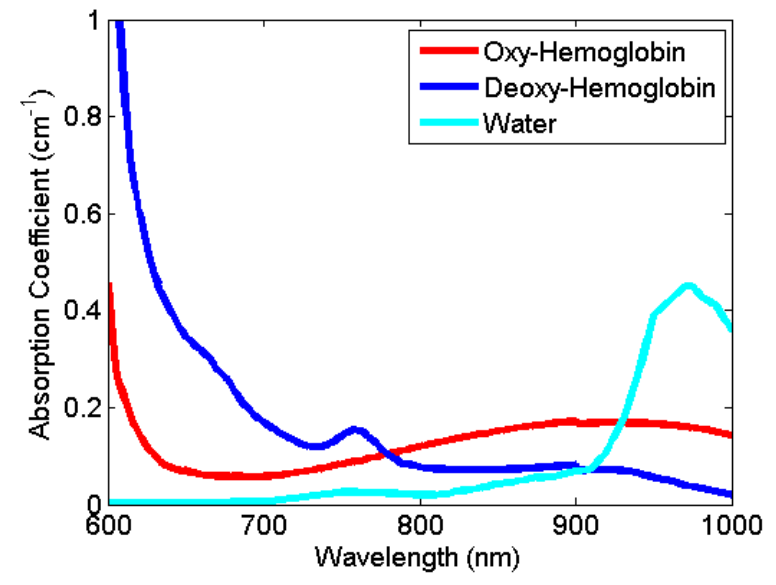

Source: http://www.scholarpedia.org/article/Near_infrared_imaging

Whole-body imaging is dependent upon both the quality of the excitation photon as well as the light emission from the fluorophore. NIR lasers produce a high-quality, focused excitation light needed for in depth imaging. When used to excite NIR fluorophores that have high extinction coefficients, low non-specific binding to tissue, and good quantum yields, the quality and sensitivity of the image is significantly enhanced (Licha and Olbrich, 2005).

Currently, we have utilised NIR instruments developed by LiCor, Inc. for our in vivo detection studies (Hale et al., 2010). The sensitivity of NIR imaging and availability of NIR fluorophore-labelled ligands led us to begin preliminary investigations as to see if the technology could be used to follow and identify botulinum neurotoxin (BoNT) in vivo localisation sites (Hale et al., 2010). A detoxified BoNT/A recombinant protein was labelled with the $800 \mathrm{CW}$ NIR dye (BoNT/A-800) and injected into mice using several different routes. The mice were then scanned $(-1 \mathrm{~h}, 1.5 \mathrm{~h}, 5 \mathrm{~h}$, and $24 \mathrm{~h})$ using the pearl imaging system (LiCor Inc., Lincoln, NE). Images of the scans showed that the localisation of fluorescence was initially dependent upon the route of injection. When injected into the muscle, fluorescence remained localised in the muscle, indicating that BoNT/A does not leave the injection site. When BoNT/A-800 was given by oral, intravenous, intranasal, or intraperitoneal routes, the fluorescence in the body varied, but at $24 \mathrm{~h}$, fluorescence was localised in the upper abdomen. The fluorescence was visible in the upper abdomen for at least $48 \mathrm{hr}$, indicating that the toxin may bind preferentially to sensory nerves in the diaphragm.

Although our studies indicate that NIR imaging provides a method to track BoNT in the whole body, further investigations are needed in which the dose, route of injection, and time course are varied. Sensitivity becomes an issue in toxin detection because the small toxin dose required to cause a response may be below the limit of detection. Further 
studies with injection of BoNT/A-800 indicate that in vivo detection of at least $50 \mathrm{ng}$ is possible (unpublished data). If combined with methods that enhance the signal, such as a FRET-based assay, tracing a lower dose of toxin $(\sim 10-30 \mathrm{ng})$ should be possible. The ability to monitor a toxin's movement through the body will further our understanding how the toxin causes damage, and will help to characterise those sites that may provide the most effective therapeutic intervention.

While our studies are preliminary, NIR imaging is currently being used the visualise tissue damage and abnormalities within the body and has been successful for tracking tumour metastasis, mapping cannabinoid and dopamine receptors in the brain, and developing animal models for Alzheimer's disease (Kearn, 2004; Skoch and Bacskai, 2004; Kovar et al., 2006; Raymond et al., 2008). In addition to ligand-conjugated to NIR fluorophores, a FRET-based assay has been developed to measure apoptosis in tumours (Messerli et al., 2004). When the optical-quenched NIR-caspase-1 substrate is injected i.v., the substrate remains quenched until cleaved by caspase 1. The substrate is cleaved, releasing the signal from its quenched state. The ability to measure apoptosis in a tumour provides an important step, not only from the ability to follow tumour death after treatment, but also the ability to develop more sensitive probes for detection within the whole body.

In addition to ligand-based detection, two other NIR-based technologies show promise for identifying tissue damage in vivo. One technology relies upon non-toxic contrasting agents that fluoresce in the NIR spectrum range. The contrasting agent is injected into the body, usually by an intravenous route, and fluorescence is monitored over time (Kim et al., 2004; Longmire et al., 2008). Unless bound to tissue, the contrasting agent is rapidly removed from the body which permits the contrasting agents to monitor various systems and determine if they appear normal. Indocyanine green (ICG), for example, is a polymethine dye that rapidly binds to $98 \%$ of plasma proteins; unbound dye is rapidly removed via the liver. The ICG-bound plasma proteins fluoresce visualises movement through the vasculature and assess tissue damage within the body. ICG is FDA-approved and has been highly successful in identifying vascular damage in various organs and tissues. Modifications of ICG or development of new contrast agents will provide methodology to assess other forms of injury such as muscle damage or bone loss. Because imaging may be viewed in real-time, contrast agents will provide surgeons with the ability to monitor vascular damage during and after surgical procedures. Such real-time measurements will allow surgeons the ability to visualise the effects that the procedure has upon the tissues. NIR contrasting agents will enable surgeons to 'see' tissues during procedures such as angioplasty or other laproscopic procedures.

Another type of imaging, functional NIR (fNIR) is non-invasive and may eventually replace other more cumbersome and expensive whole body tomographies such as MRI and PET. The technology was originally developed by Briton Chance (Chance et al., 1998) for the purpose of characterising brain function in relation to cogitative tasks being performed. The photon-generating source is placed on the head so that the detecting device measures NIR light absorbance of hemoglobin with and without oxygen (Flaumenhaft et al., 2007; Tamura et al., 1997). Brain activity measured in relation to blood flow and oxygen levels have been able to correlate changes in the NIR absorbance with brain damage caused by injury or stroke. In addition to brain activity studies, fNIR is being developed to detect breast cancer or other abnormalities and results appear to be promising, although images are not yet sharp enough for sensitive detection. Further 
research and improvements will enable NIR tomography to provide a new technique for cancer screening and perhaps provide more sensitive detection.

In summary, NIR technology offers a wide array of methods to identify toxin movement and to characterise tissue damage caused by a variety of agents. NIR optical imaging provides non-invasive and rapid detection methods that can be used to further understand how the body responds to intoxication, infection, or injury.

\section{Acknowledgements}

The views expressed in this article are those of the author and do not necessarily reflect the official policy or position of the Department of the Army, the Department of Defense, or the US Government.

\section{References}

Chance, B., Anday, E., Nioka, S., Zhou, S., Hong, L., Worden, K., Li, C., Murray, T., Ovetsky, Y., Pidikiti, D. and Thomas, R. (1998) 'A novel method for fast imaging of brain function, non-invasively, with light', Opt Express, Vol. 2, No. 10, pp.411-423.

Flaumenhaft, R., Tanaka, E., Graham, G.J., De Grand, A.M., Laurence, R.G., Hoshino, K., Hajjar, R.J. and Frangioni, J.V. (2007) 'Localization and quantification of platelet-rich thrombi in large blood vessels using near-infrared fluorescence imaging', Circulation, Vol. 115, No. 1, pp.84-93.

Frangioni, J.V. (2003) 'In vivo near-infrared fluorescence imaging', Curr. Opin. Chem. Biol., Vol. 7, No. 5, pp.626-634.

Hale, M.L., Riding, S. and Singh, B.R. (2010) 'Near-infrared imaging of Balb/c mice injected with a detoxified botulinum neurotoxin A', The Botulinum J., No. 1.

Kearn, C.S. (2004) 'Immunofluorescent mapping of cannibinoid CB1 and dopamine D2 receptors in the mouse brain', Li-Cor Biosciences, available at http://www.licor.com.

Kim, S., Lim Y.T., Soltesz, E.G., De Grand, A.M., Lee, J., Nakayama, A., Parker, J.A., Mihaljevic, T., Laurence, R.G. and Dor, D.M. (2004) 'Near-infrared fluorescent type II quantum dots for sentinel lymph node mapping', Nat. Biotechnol., Vol. 22, No. 1, pp.93-97.

Kovar, J.L., Johnson, M.A., Volcheck, W.M., Chen, J. and Simpson, M.A. (2006) 'Hyaluronidase expression induces prostate tumor metastasis in an orthotopic mouse model', Am. J. Pathol., Vol. 169, No. 4, pp.1415-1426.

Licha, K. and Olbrich, C. (2005) 'Optical imaging in drug discovery and diagnostic applications', Adv. Drug Deliv. Rev., Vol. 57, No. 8, pp.1087-1088.

Longmire, K., Choyke, P.L. and Kobayashi, H. (2008) 'Dendrimer-based contrast agents for molecular imaging', Cur. Topics in Medicinal Chem., Vol. 8, No. 14, pp.1180-1186.

Messerli, S.M., Prabhakar, S., Tang, Y., Shah, K., Cortes, M.L., Murthy, V., Weissleder, R., Breakefield, X.O. and Tung, C.H. (2004) 'A novel method for imaging apoptosis using a caspase-1near-infrared fluorescent probe1', Neoplasia, Vol. 6, No. 2, pp.95-105.

Olive, D.M. (2006) 'Near infrared technology and optical agents for molecular imaging', Li-Cor Biosciences, available at http://www.licor.com.

Raymond, S.B., Skoch, J., Hills, I.D., Nesterov, E.E., Swager, T.M. and Bacskai, B.J. (2008) 'Smart optical probes for near-infrared fluorescence imaging of Alzheimer's disease pathology', Eur. J. Nucl. Med. Mol. Imaging, Vol. 35, No. 1, pp.S93-S98. 
Skoch, J. and Bacskai, B.J. (2004) 'The Li-COR Odyssey as a near infrared imaging platform for animal models of Alzheimer's disease', Li-Cor Biosciences, available at http://www.licor.com/bio/PDF/MassGen.pdf.

Tamura, M., Hoshi, Y. and Okada, F. (1997) 'Localized near-infrared spectroscopy and functional optical imaging of brain activity', Philos. Trans. R. Soc. Lond. B. Biol. Sci., Vol. 352, No. 1345, pp.737-742. 